\title{
On the capabilities of survey telescopes of moderate size
}

\author{
V. Yu. Terebizh ${ }^{1,2 *}$ \\ ${ }^{1}$ Crimean Astrophysical Observatory, Nauchny, Crimea 298409 \\ ${ }^{2}$ Institute of Astronomy RAN, Moscow 119017
}

February 09, 2016

\begin{abstract}
To explore capabilities of moderate-size optical telescopes in surveys, the set of 9 new wide-field designs having apertures up to $1 \mathrm{~m}$ is considered. All but one systems have angular field of view in a range $3.5^{\circ}-10^{\circ}$ and flat focal surface; the field of the last system is $45^{\circ}$ in diameter at the $0.5 \mathrm{~m}$ aperture and spherical focal surface. The complete description of the optical layouts is given in the Appendix. Relations between the expected limiting magnitude, survey speed and exposure time allow to choose the system that is most suitable for a particular task of observations. In principle, a single wide-field telescope with the aperture of approximately $1 \mathrm{~m}$ can detect objects brighter than $22.5^{m}$ over the entire hemisphere within one night, however, the reliability of acquired data can be significantly increased by using a hierarchic observational network comprised of telescopes with optimized parameters.
\end{abstract}

\section{Introduction}

A number of important astrophysical problems necessitates continuous registration of all objects of the sky brighter than about $23^{m}$ in the visible

*E-mail: valery@terebizh.ru 
waveband. To estimate the required survey speed $S$, measured in square degrees per second $\left(\mathrm{deg}^{2} / \mathrm{sec}\right)$, we imply that one needs to cover a sky area of $10^{4} \mathrm{deg}^{2}$ within 3 hours. This area is a little smaller than the entire hemisphere visible above the horizon and free of absorption in the Milky Way and Earth's light pollution at large zenith distances. This gives us the value of $S \simeq 1 \mathrm{deg}^{2} / \mathrm{sec}$ and makes the problem quite non-trivial. Indeed, the field of view of a so-called classical telescope (parabolic primary mirror plus hyperbolic secondary mirror) is only several arc minutes wide. Thus, one needs to acquire about $10^{6}$ images to cover the considered sky area, which is unrealistic even with several telescopes. Within this scope even RitcheyChretien telescopes, recently considered wide-field, fail to solve the problem: the typical field of a Ritchey-Chretien telescope does not exceed 20', which might reduce the number of images, mentioned above, by only an order of magnitude.

In this regard, we would naturally turn to a remarkable Bernhardt Schmidt (1930) system, whose modified versions reach a field of about $10^{\circ}$ in diameter. Astronomers had used these systems for over fifty years while the photographic plates were light detectors: one had to bend the plate to match the curved focal surface of the Schmidt camera (its curvature radius is about the effective focal length). Meanwhile, the majority of modern detectors are flat. One can achieve the flat field either by complicating the optical system or by making the field faceted with small field-flatteners. The last option has been applied to the Kepler telescope, which has the aperture of $95 \mathrm{~cm}$ and the field area of $115 \mathrm{deg}^{2}$. Its detector consists of 21 pairs of ordinary $59 \mathrm{~mm}$ $\times 28 \mathrm{~mm}$ CCDs covered by sapphire field-flattening lenses. Obviously, this way is feasible now only in unique projects, so the most designs discussed below have flat focal surface. The exception is an all-spherical design with a $45^{\circ}$ field - the particular implementation of a system, which was proposed recently by the author (Terebizh 2015, 2016).

The first step towards simplification of the Schmidt camera its author made himself by testing in 1934 a model with three spherical lenses instead of the aspheric corrector (see Wachmann 1955; Busch et al. 2013). In fact, all the subsequent wide-field catadioptric telescopes - the systems by Richter and Slevogt (1941), Schmidt-Houghton (Houghton 1942, 1944), Hawkins and Linfoot (1945), Baker (1962) and $\Omega_{2-3}$ (Terebizh 2007a,b) - are the successors of the two generic systems, invented by Schmidt. Modern versions of these systems provide angular field up to $10^{\circ}$ at flat focal surface and aperture reaching $1 \mathrm{~m}$ (Terebizh 2011). 
Another approach, implying a lens corrector mounted in the vicinity of the focus of a large aspheric mirror, was introduced by Sampson (1913), Ross (1935) and Wynn (1968). Within this approach one cannot achieve a field, comparable with that of a mid-size catadioptric system, but the large aperture diameter allows detecting faint objects. The modern types of prime-focus correctors were proposed by Terebizh (2003) and Saunders et al. (2014).

This paper was initiated by researchers questions that arise in the development of survey projects, first of all: what an optical system is best suited at the specified survey depth and speed? We compare the relevant efficiency of various catadioptric designs with the angular field in a range of $3.5^{\circ}-10^{\circ}$ (flat focal surface) and the $45^{\circ}$-design with a spherical focal surface (Fig. 1). Apertures of catadioptric designs are in the range from $0.4 \mathrm{~m}$ up to $1.0 \mathrm{~m}$. A $20-\mathrm{cm}$ refractive lens with a $15^{\circ}$ angular field and flat focal surface was added for comparison 1 .

To be specific, the linear diameter $B$ of the flat field has been adopted the same for all the designs, $134.5 \mathrm{~mm}$, which coincides with the length of the diagonal CCD STA 1600 of Semiconductor Technology Associates. Thus, we leave aside the telescopes with huge mosaic detectors. Obviously, the latter provide larger angular field of view, however, our goal now is to maintain the uniformity of results.

Observations with the curved focal surface need special discussion, which we give in Section 2.2.

Since one should take into account the specifications of light detectors when designing an optical system, we briefly touch on issues related to the matching the telescope's and detector's resolving power.

\section{Optical layouts}

\subsection{Flat focal surface}

The set of flat-field systems that we consider hereafter includes a telescope with a prime-focus corrector, a corrected Cassegrain system, a Schmidt camera, a Schmidt-Houghton telescope, a modified Richter-Slevogt telescope (Terebizh 2001), a version of the Amon, Rosin and Jackson camera (Amon

\footnotetext{
${ }^{1}$ In calculations, we used the Zemax optical program (ZEMAX Development Corporation, U.S.A.).
} 
et al. 1971), $\Omega_{2}$ and $\Omega_{3}$ systems (Terebizh 2007a,b), and a lens objective to compare its efficiency with that of catadiopric systems (Fig. 1). Basic optical layouts of these systems have long been known, almost all of them are implemented. New versions were designed for this paper, which provide the widest possible field of view given the image quality and size of the detector.
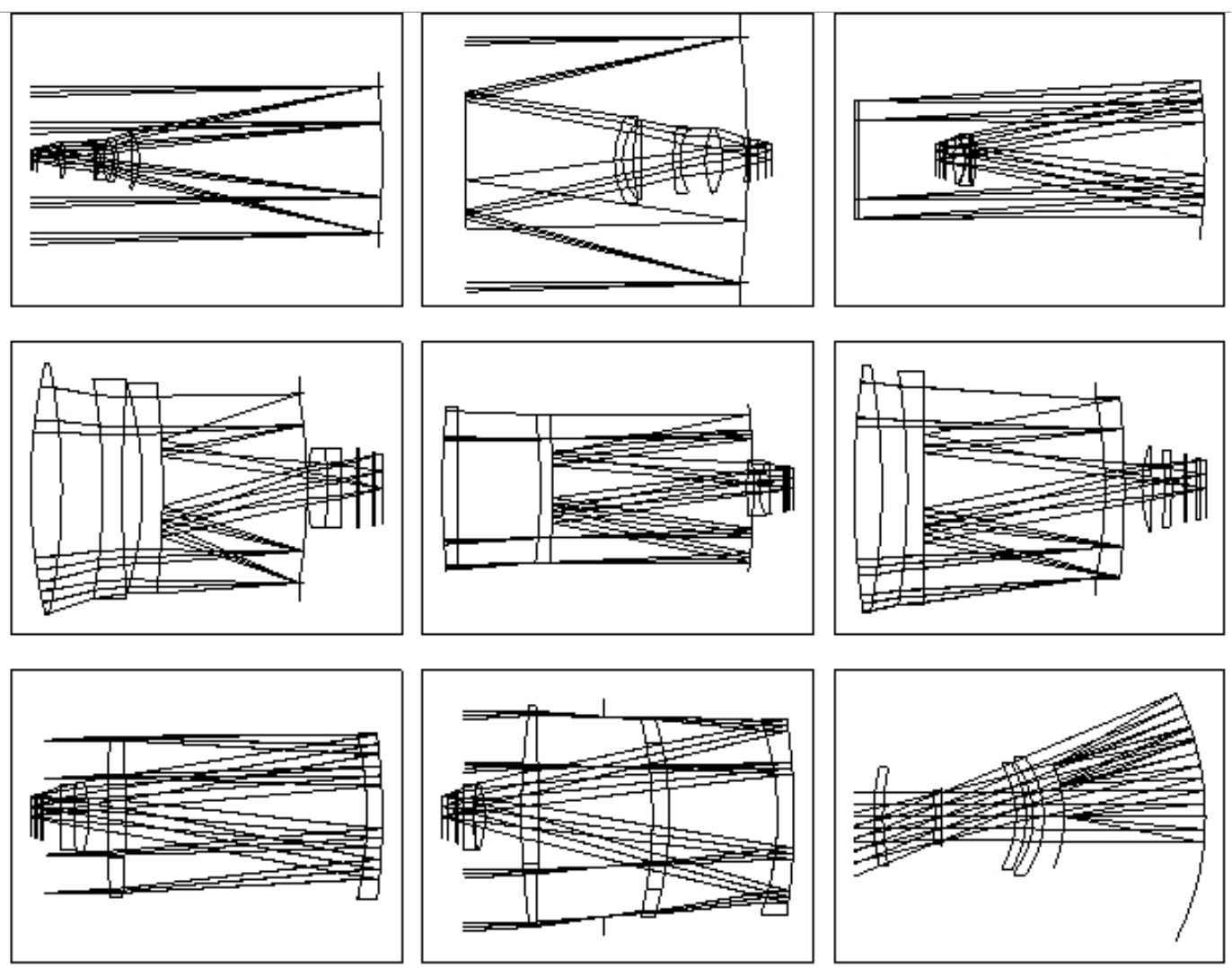

Figure 1: Optical layouts of survey telescopes discussed in this paper. First row, from left to right: VT-56y, -112m, -110f. Second row: VT-78e, -77i, -98v. Third row: VT-102j, -60g, -119j.

General characteristics of the flat-field telescopes are given in Table 1, namely: the sequence number; number according to the author's catalogue; entrance pupil diameter $D(\mathrm{~mm})$; angular diameter of the field of view $2 w$ (degree); effective focal length $F(\mathrm{~mm})$; spectral range used in calculations $(\mu \mathrm{m})$; fraction of unvignetted rays $U$ on the optical axis and on the edge of a 
Table 1: General characteristics of telescope designs a with flat (No. 1 - 9) and curved (No. 10) focal surface.

\begin{tabular}{rrrrrcccccr}
\hline No. & $\begin{array}{r}\text { VT- } \\
\text { No. }\end{array}$ & $\begin{array}{r}\mathrm{D} \\
(\mathrm{mm})\end{array}$ & $2 w^{\circ}$ & $\begin{array}{r}\mathrm{F} \\
(\mathrm{mm})\end{array}$ & $\begin{array}{c}\text { Waveband } \\
(\mu \mathrm{m})\end{array}$ & $\mathrm{U}$ & $\begin{array}{c}D_{80} \\
(\mu \mathrm{m})\end{array}$ & $D_{80}^{\prime \prime}$ & $\begin{array}{c}\Gamma \\
(\mathrm{H})\end{array}$ & $\begin{array}{r}\mathrm{L} \\
(\mathrm{mm})\end{array}$ \\
\hline 1 & $56 \mathrm{y}$ & 1000 & 3.5 & 2183 & $0.40-0.85$ & $0.85-0.85$ & $6.5-7.0$ & $0.61-0.66$ & 1.90 & 2299 \\
2 & $112 \mathrm{~m}$ & 1000 & 3.5 & 2189 & $0.45-0.85$ & $0.69-0.69$ & $10.6-11.4$ & $1.0-1.1$ & 1.28 & 1236 \\
3 & $110 \mathrm{f}$ & 500 & 7.5 & 1025 & $0.42-0.82$ & $0.75-0.75$ & $8.2-10.8$ & $1.6-2.2$ & 0.71 & 1500 \\
4 & $78 \mathrm{e}$ & 400 & 10.0 & 764 & $0.45-0.85$ & $0.72-0.51$ & $8.9-10.9$ & $2.4-2.9$ & 0.47 & 679 \\
5 & $77 \mathrm{i}$ & 500 & 5.0 & 1538 & $0.45-0.85$ & $0.74-0.57$ & $10.4-13.9$ & $1.4-1.8$ & 0.47 & 1132 \\
6 & $98 \mathrm{v}$ & 500 & 7.0 & 1093 & $0.45-0.85$ & $0.68-0.56$ & $7.8-10.3$ & $1.5-1.9$ & 0.64 & 829 \\
7 & $102 \mathrm{j}$ & 525 & 7.0 & 1094 & $0.43-0.85$ & $0.79-0.79$ & $8.0-9.6$ & $1.5-1.6$ & 0.87 & 1207 \\
8 & $60 \mathrm{~g}$ & 700 & 7.0 & 1091 & $0.45-0.85$ & $0.89-0.89$ & $8.4-10.5$ & $1.6-2.0$ & 1.58 & 1159 \\
9 & $101 \mathrm{k}$ & 200 & 15.0 & 504 & $0.45-0.85$ & $1.0-1.0$ & $8.5-10.1$ & $3.5-4.1$ & 0.18 & 712 \\
10 & $119 \mathrm{j}$ & 500 & 45.0 & 1358 & $0.45-0.85$ & - & $8.2-9.5$ & $1.2-1.4$ & - & 3359 \\
\hline
\end{tabular}

Telescopes brief descriptions:

1. Prime focus corrector.

2. Corrected Cassegrain.

3. Schmidt with a 3-lens corrector-flattener.

4. Schmidt (1934) - Houghton (1942, 1944).

5. Modified Richter-Slevogt.

6. Richter-Slevogt with a Mangin-type primary (Amon et al. 1971).

7. Double-pass singlet corrector with a Mangin primary $\left(\Omega_{2}\right.$, Terebizh 2007a).

8. Double-pass 2-lens corrector with a Mangin primary $\left(\Omega_{3}\right.$, Terebizh 2007a).

9. Refractive lens.

10. All-spherical system with a 4-lens corrector (Terebizh 2015).

field; diameter $D_{80}$ of a circle that contains $80 \%$ of the energy in a polychromatic star's image ( $\mu \mathrm{m}$ and arc seconds); sky survey rate $\Gamma$ (Herschels), and the total length of the system from the first optical surface to the detector $L(\mathrm{~mm})$. The focal ratios are presented below in Table 2 .

The term 'sky survey rate' and the corresponding measurement unit 'Herschel' are explained in Section 5. All systems have been optimized in the integral light within the waveband boundaries specified in Table 1, but they can be used in a wider spectral range. The common linear obscuration coefficient $\eta$ is related to the parameter $U$ as $U=1-\eta^{2}$, both depending on the field angle. The effective aperture diameter $D_{e}=D U^{1 / 2}$. The image diameter $D_{80}$ corresponds to the waveband specified in Table 1 ; it is obvious 


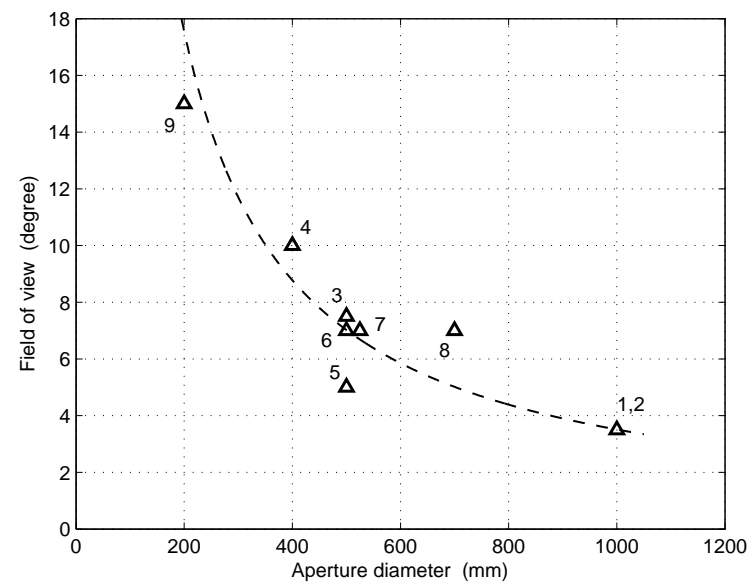

Figure 2: Relation between the diameters $D(\mathrm{~mm})$ of flat-field systems in Table 1 and their angular field of view $2 w(\mathrm{deg})$. Dashed line corresponds to the approximate equation $2 w=3510 / D$.

that by using of narrow-band filters one can improve image quality.

We do not consider some attractive systems providing good images in the field up to $3.5^{\circ}$, in particular, the three-mirror anastigmat by Dietrich Korsch $(1972,1977)$ and the Mersenne-Schmidt telescope by Maurice Paul (1935; see Willstrop 1984 as well). The reason is that for the aperture less than $1 \mathrm{~m}$ one can attain the same image quality and field size with simpler optics and lower obscuration. In contrast, in the case of large telescopes, where the fullaperture field correctors cannot be applied, the systems mentioned above provide maximal depth of the survey. Thus, the layouts by Korsch and Paul were selected, respectively, for the space telescope SNAP of $2 \mathrm{~m}$ aperture with the field of $1.5^{\circ}$ diameter and for the $8.4 \mathrm{~m}$ ground-based telescope LSST with the field $3.5^{\circ}$.

Full descriptions of all the systems are given in the Appendix to this paper.

Fig. 2 gives an idea of the telescope aperture $D$ and the angular field of view diameter $2 w$ for systems listed in Table 1 . As in the much more extensive set of existing telescopes discussed by Terebizh (2011), the field of view size is, to a first approximation, inversely proportional to the aperture 
diameter: $2 w^{\circ} \simeq 3.51 / D_{m}$. This dependence arises from a simple relation

$$
B \simeq D \phi \cdot 2 w^{\circ} / 57.3
$$

given that the linear size of the detector $B$ and focal ratio $\phi \equiv F / D$ are changing insignificantly. In our case, this condition is satisfied, because the detector diagonal is fixed and $\phi$ are close to the mean value $\langle\phi\rangle \simeq 2.20$ (see Table 2).

Since aberrations of optical systems grow rapidly with increasing of their speed, a small spread in $\phi$ is specific for wide-field telescopes. Thus, the relation (1) clearly shows the need to increase the size of the detectors for large survey telescopes.

The primary mirrors of the two first telescopes from Table 1 have aspheric surfaces. The main problem that arises when manufacturing such systems is not as much concerned with the maximum deviation of the mirror's surface from sphere but with the asphericity gradient $G(\mu \mathrm{m} / \mathrm{mm})$, i.e., the rate at which the deviation changes along the radial coordinate. An approximate expression for the maximum asphericity gradient $G_{\max }$ of a conic section as a function of its diameter $D$, curvature radius at vertex $R_{0}$ and eccentricity $\varepsilon$ is:

$$
G_{\max } \simeq 31.25 \varepsilon^{2}\left(D /\left|R_{0}\right|\right)^{3}, \quad \mu m / m m
$$

(Terebizh 2011). Usually, the asphericity gradient of the primary mirror does not exceed $0.6 \mu \mathrm{m} / \mathrm{mm}$. For the fastest existing wide-field telescopes this value reaches $1.5 \mu \mathrm{m} / \mathrm{mm}$ (see Fig. 4 in the paper mentioned above). Here, the values of $G_{\max }$ are $0.45 \mu \mathrm{m} / \mathrm{mm}$ and $0.58 \mu \mathrm{m} / \mathrm{mm}$ for the systems No. 1 and No. 2, respectively, i.e. they are relatively small.

Although a corrected Cassegrain system is, in some essential respects, inferior to a system with the prime-focus corrector, its compactness may play the decisive role if necessary to make a number of identical instruments.

In this regard, it is worth adding that the image quality in corrected Cassegrain systems depends weakly on the shape of the secondary mirror. In those cases, where the squared eccentricity of this mirror reaches values of the order of 20 or even higher (for example, in PAN-STARRS; Hodapp et al. 2004), the gain in the image quality is totally smeared out by the manufacturing problems and severe tolerances in operation. On the contrary, tolerances are much more loose for systems with spherical secondary mirrors, which significantly increases the productivity of observations (an obvious example is GEODSS; Jeas 1981). The reason for such a small influence of 
the secondary mirror's shape in wide-field telescopes is clear: this mirror should be equally optimal for the light beams falling on it at very different angles, and this is possible only when the secondary mirror is close to a sphere.

\subsection{Spherical focal surface}

The general description of an all-spherical telescope with extremely wide field of view and spherical focal surface was given by Terebizh $(2015,2016)$. A few examples and the corresponding discussion were given in those papers. We propose here one more example of an $f / 2.7$ system with an aperture of $500 \mathrm{~mm}$ and a $45^{\circ}$ angular field of view. It is included in Table 1 at No. 10; see also Fig. 1 and Fig. 3 for the optical layout and spot diagram; Appendix includes a complete set of parameters. Since the parameters $U$ and $\Gamma$ depend on the shape of detector, their values are left undefined. Generally speaking, the lenses can be made of arbitrary types of glass; in this case we choose the fused silica. In spite of a huge field size, the system provides good images: the $D_{80}$ image diameter in the polychromatic waveband $0.45-0.85 \mu \mathrm{m}$ varies across the field in the range $1.2^{\prime \prime}-1.4^{\prime \prime}$, whereas the Airy disc diameter is $0.64^{\prime \prime}$.

Evidently, the radical expanding of angular field of high-quality images is a consequence of the transition to a purely spherical optics. Just this feature provides the real point symmetry of the system that is limited only by inevitable vignetting on the aperture stop. The second feature of the system that provides almost complete absence of chromaticity, is the afocality of the 4-lens corrector: it works at a $f / 87$ speed. Finally, the third property necessary for ensuring wide field is the close proximity of the entrance pupil to the aperture diaphragm; their separation is only $31 \mathrm{~mm}$ in a case under consideration.

As mentioned in the Introduction, the main problem with such systems is the need to work with the curved focal surface. The following ways seem to be preferred now in this regard: i) the use of large detectors with a curved surface; $i i$ ) applying the long-known technology based on a plurality of delicate waveguides with a curved in aggregate input faced to the focal surface; iii) using a small flat detectors equipped with a flattening optics.

The principal issues and examples of curved detectors were discussed by Iwert and Delabre (2010), Iwert et al. (2012); the first of these papers includes a photograph of a curved detector with size of $60 \mathrm{~mm} \times 60 \mathrm{~mm}$ 


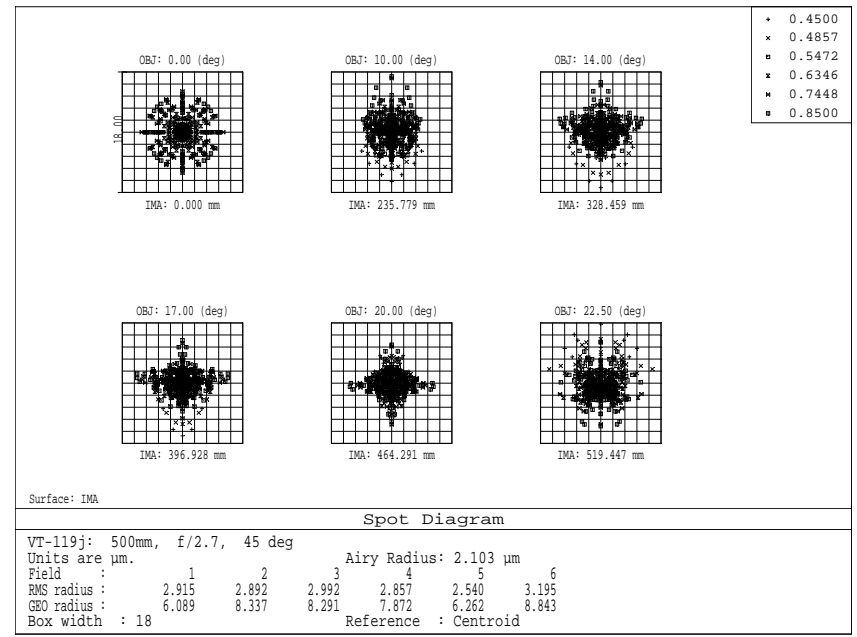

Figure 3: Spot diagram of VT-119j design in the polychromatic waveband $0.45-0.85 \mu \mathrm{m}$. The field angles are $0^{\circ}, 10^{\circ}, 14^{\circ}, 17^{\circ}, 20^{\circ}$ and $22.5^{\circ}$. Airy disc diameter is $4.2 \mu \mathrm{m}$, box width is $18 \mu \mathrm{m} \simeq 2.7^{\prime \prime}$.

and curvature radius $500 \mathrm{~mm}$. There are also working devices of this type. In particular, curved detector has been implemented in the DARPA $3.5 \mathrm{~m}$ Space Surveillance Telescope (Blake et al. 2013). It is important to note that individual detectors of any form can be placed, both continuously and discretely, on the curved focal surface according to the shape of the studied area of the sky.

The second option, being considered in a modern context, involves a number of technological problems. One should expect that basically these problems will be solved within the framework of the announced by European Space Agency in 2013 program, which provides a solution for mapping a curved image field onto a flat imaging detector array.

We mentioned the third option in Sec. 1 in connection with the Kepler telescope. In a case of VT-119j, the curvature radius of the focal surface is rather large, $1329 \mathrm{~mm}$, so an additional flattening optics can be made of a single lens. Let us consider, for example, a flat detector of format $30 \mathrm{~mm} \times$ $30 \mathrm{~mm}$, i.e., with a diagonal length $42.4 \mathrm{~mm}$. If we keep the quality of central images, then their corner diameter will be about $50 \mu \mathrm{m}$. We can made the blur of spots across the field less than $23 \mu \mathrm{m}$ by shifting the detector at $0.1 \mathrm{~mm}$. Finally, the image quality is restored completely, when a weak lens made of fused silica is installed in front of the detector. Since the lens radii 
of curvature are of about $300 \mathrm{~mm}$ and $200 \mathrm{~mm}$, one can simply use it as the detector window.

Thus, in the case under consideration, the third option is the most straightforward.

\section{Sampling factor}

As one can see, the value of $D_{80}$ characterizes the image quality provided by a telescope alone; to distinguish $D_{80}$ from similar quantities we designate it hereafter as $\beta_{t e l}$. For the telescopes considered in this paper, it varies from $0.6^{\prime \prime}$ up to $2.9^{\prime \prime}$, i.e., has the same order of magnitude as the atmospheric blurring $\beta_{\text {atm }}$. For our purposes, it is enough to accept that the angular diameter of a star image due to these two factors is

$$
\beta=\sqrt{\beta_{a t m}^{2}+\beta_{t e l}^{2}} .
$$

Accepting constant $\beta_{a t m}=1.5^{\prime \prime}$ and $\beta_{\text {tel }}$ according to Table 1 , we obtain the resulting values of image quality $\beta$ shown in the fourth column of Table 2 . Let us accept also the linear size of the detector's pixel equal to $9 \mu \mathrm{m}$, as in the CCD STA 1600. The corresponding angular sizes of pixels are given in the fifth column of Table 2 .

Among the set of parameters that define the area of application of a telescope, an important role plays the sampling factor $\chi$ - the ratio of the diameter of the star image to the size of the pixel:

$$
\chi \equiv \beta / p,
$$

where both $\beta$ and $p$ are either angular or linear. According to the well-known sampling theorem by V. Kotel'nikov and C. Shannon (see, e.g., Press et al. 1992 , p. 500), the retaining of the entire spectrum of spatial frequencies of a continuous image at sampling requires the discretization step, which not exceeds $\delta x_{c} \equiv 1 /\left(2 f_{c}\right)$, where $f_{c}$ is the cutting frequency above which the power spectrum of the continuous object can be considered negligible. In typical astronomical applications $\delta x_{c}$ is approximately equal to half a radius of the star's image. Thus, one usually should have at least 4 pixels covering the diameter of a star image (i.e. $\chi \simeq 4$ ). Taking into account random fluctuations of flux, this value is usually increased to 8 in precise photometric measurements ( $\chi \simeq 7$ for the Kepler space telescope). On the other hand, 
Table 2: Focal ratio $\phi \equiv F / D$ and sampling factor $\chi$ at the atmosphere blurring $1.5^{\prime \prime}$ and linear pixel size $9 \mu \mathrm{m}$.

\begin{tabular}{cccccc}
\hline $\begin{array}{c}\text { System } \\
\text { No. }\end{array}$ & $\phi$ & $\begin{array}{c}\text { Scale } \\
\mu \mathrm{m} /{ }^{\prime \prime}\end{array}$ & $\beta^{\prime \prime}$ & $p^{\prime \prime}$ & $\chi$ \\
\hline 1 & 2.18 & 10.6 & 1.62 & 0.85 & 1.9 \\
2 & 2.19 & 10.6 & 1.83 & 0.85 & 2.2 \\
3 & 2.05 & 4.97 & 2.42 & 1.81 & 1.3 \\
4 & 1.91 & 3.71 & 3.05 & 2.43 & 1.3 \\
5 & 3.08 & 7.46 & 2.16 & 1.21 & 1.8 \\
6 & 2.19 & 5.30 & 2.27 & 1.70 & 1.3 \\
7 & 2.08 & 5.30 & 2.16 & 1.70 & 1.3 \\
8 & 1.56 & 5.29 & 2.34 & 1.70 & 1.4 \\
10 & 2.72 & 6.58 & 1.98 & 1.37 & 1.4 \\
\hline
\end{tabular}

in surveys, where detecting faint objects is of the primary importance, the sampling factor is reduced to $1-2$.

The $\chi$ values corresponding to the conditions we have adopted are given in the last column of Table 2. We can see that the discussed sample of telescopes exactly suites the work of search or exploratory nature.

\section{Limiting magnitude and survey speed}

Let us now consider the characteristics of wide-field telescopes that are of special interest within the scope of this paper. Namely, these are the limiting magnitude $\left(m_{\text {lim }}\right)$ and survey speed $\left(S, \operatorname{deg}^{2} / \mathrm{sec}\right)$ determined by the telescope + detector system and observational conditions. In calculations, we took into account the entrance pupil diameter, effective focal length, angular field of view, telescope transparency, fraction of unvignetted rays, bandwidth, and $\beta_{t e l}$ value. Parameters of the detector are the same for telescopes: the quantum efficiency is 0.85 events/photon and the pixel size is $9 \mu \mathrm{m}$ (CCD STA 1600). It was assumed that the noise obeys the Poisson distribution. As concerns the observational conditions, we have assumed that $\beta_{a t m}=1.5^{\prime \prime}$, sky background is $20.0^{\mathrm{m}} / \operatorname{arcsec}^{2}$, optical thickness of the atmosphere in zenith is 0.30 , object zenith angle is $40^{\circ}$, dead time is $5 \mathrm{sec}$, and the threshold signalto-noise ratio $S / N=8$. The parameter we call 'dead time' is the sum of read-out and telescope redirection time spans. The $S / N$ ratio corresponds 


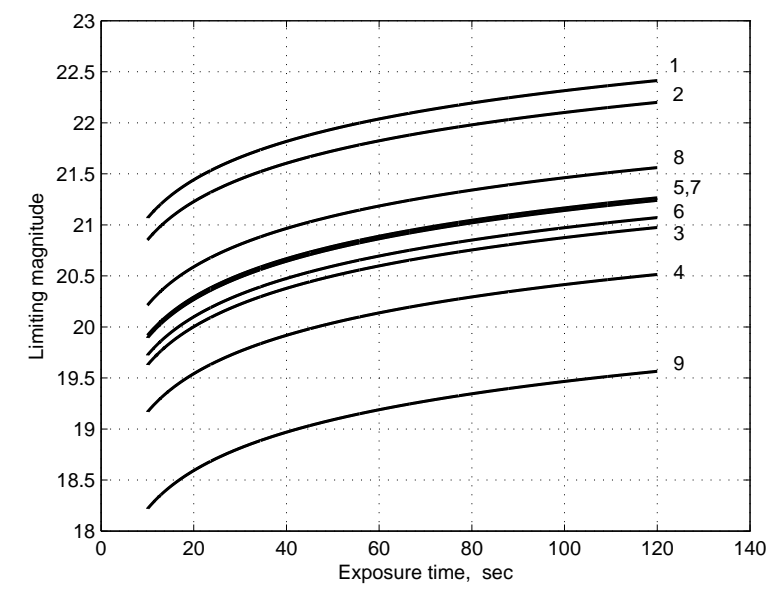

Figure 4: Limiting magnitude as a function of the exposure time for the flat-field systems in Table 1.

to the total number of pixels in detector. The variable value in our calculations is the exposure time $T$. We tried to adopt the above parameters as close as possible to their typical values. Of course, variations of initial values change the results, but not radically.

Figures 4 and 5 demonstrate resulting values of the limiting magnitude and survey speed, respectively. The first are in a quite good agreement with the estimates according to the SIGNAL package created by the team of Isaac Newton Group of Telescopes (http://catserver.ing.iac.es/signal/). However, we do not require the calculations to exactly match the real data, because our primary goal is to evaluate the comparative characteristics of various types of optical systems.

As one can see, flat-field optical systems are clearly divided into three groups: $i$ ) systems No. 1 and No. 2 ; ii) systems No. 3 - 8; iii) lens objective No. 9. This division results from the initial grouping according to $D$ and $2 w$ values (see Fig. 2), because the limiting magnitude depends primarily on the aperture diameter and does not depend on the field size, while the survey speed is proportional to the field area, which is maximal for relatively small telescopes.

Since $m_{\text {lim }}$ grows and $S$ decreases with increasing of $T$, we can expect that some their combination is independent, to a first approximation, on the exposure time. Indeed, the limiting magnitude $m_{l i m}=2.5 \log \left(D T^{1 / 2}\right)+A$, 


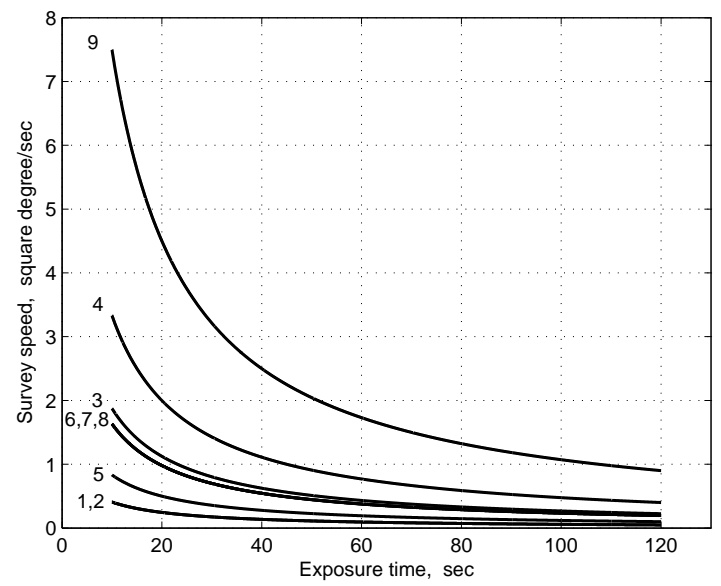

Figure 5: Survey speed as a function of the exposure time for the flat-field systems in Table 1.

where $A$ incorporates all other parameters. Taking into accound that, for relatively short dead time, the survey speed is $S \simeq(2 w)^{2} / T$, we obtain:

$$
Q \equiv m_{\text {lim }}+1.25 \log S \simeq \text { const }
$$

In our case, at $T$ in the range from $10 \mathrm{sec}$ to $90 \mathrm{sec}$, the RMS variation of $Q$ for any particular design is only 0.04 . The individual values of $Q$ vary from 19.5 for the $200-\mathrm{mm}$ refractor to 20.7 for the 1 -m system with a prime-focus corrector. Obviously, the $Q$ value depends not only on the telescope + detector system, but also on the observational conditions, so, the bigger $Q$ we reach, the more effective observational system we have. An adequate criterion of effectiveness of the telescope alone is discussed below in Section 5.

When planning a program of observations, different priority is assigned usually to the achieved stellar magnitude and survey speed. Therefore, the main problem is choosing an appropriate exposure time $T$ required to obtain the desired $m_{\text {lim }}$ and $S$ values. In turn, the adopted by an observer triplet $\left\{m_{\text {lim }}, S, T\right\}$ determines the proper choice of the telescope, namely, its optical system and the set of specifications listed in Table 1.

Let us suppose, for example, that we should provide $S \sim 1 \mathrm{deg}^{2} / \mathrm{sec}$, as mentioned in the Introduction, and the limiting magnitude $m_{\text {lim }} \simeq 20.5$. Fig. 4 shows that telescopes No. 1 and No. 2 are too big for this purpose, 
because the needed exposure time is short in comparison with the dead time. The required limiting magnitude can be achieved by using systems No. 3, $5-8$ at quite an appropriate exposure time of $25-50$ sec. However, the further consideration of Fig. 5 excludes the system No. 5 (modified RichterSlevogt), because it provides too small field. The system No. 3 (Schmidt camera) contains the corrector with a complicated aspheric surface; besides, to achieve good image quality one should use an expensive and not simple in making glass. These considerations impel us to opt for one of the allspherical telescopes with simple types of glass No. 6 (Amon et al.), No. 7 $\left(\Omega_{2}\right)$ or No. $8\left(\Omega_{3}\right)$. The final choice requires the detailed discussion of more subtle properties of these systems.

To show the essential conditionality of choosing a proper optical layout, we note that system No. 4 (all-spherical Schmidt-Houghton with a three-lens input corrector) is clearly preferable at necessity to achieve the same survey speed of $S \sim 1 \mathrm{deg}^{2} / \mathrm{sec}$ but $m_{\text {lim }} \sim 20.0$ for about $40 \mathrm{sec}$ exposure. A number of modified telescopes of this type were made within the last decade (Terebizh 2011).

Naturally, only 1-meter telescopes No. 1 and No. 2 allow detecting the faint objects in the range of $21.5^{m}-22.5^{m}$. The difference in the limiting magnitude between these two systems is $\sim 0.21^{\mathrm{m}}$; it equally results from a lower obscuration and better image quality provided by the prime-focus corrector. Both of these factors are typical for the compared systems; the installing of the secondary mirror only allows achieving compactness of the telescope at a predetermined effective focal length.

We do not include the effect of stray light and direct exposure of detector by sky background, because these factors are highly dependent not only on the optical scheme of the telescope, but also on its structure. Evidently, in this respect the telescope with a prime-focus corrector again clearly superior to systems such as the corrected Cassegrain. In practice, the need to install a complicated system of baffles in a wide-field Cassegrain design reduces both limiting magnitude and size of the field.

If necessary, the limiting magnitude of a survey telescope can be somewhat increased by reducing its angular field of view but leaving unchanged the linear dimensions of the detector. This increases the focal length of the telescope, and, as a consequence, reduces aberrations, improves image quality and reduces the contribution of the sky background in a pixel of smaller angular size.

As regards the design No. 10 with a field of $45^{\circ}$ in diameter, the corre- 
sponding limiting magnitude and survey speed depend on the relative area of detectors in the focal surface. Let us suppose, for example, that a strip of sizes $45^{\circ} \times 4^{\circ}(\sim 1000 \mathrm{~mm} \times 95 \mathrm{~mm})$ is occupied by detectors. Then, the fraction of unvignetted rays $U \simeq 0.89$, which gives the equivalent aperture diameter $D_{e} \simeq 472 \mathrm{~mm}$. Thus, the limiting magnitude for the system No. 10 is approximately equal to those for the system No. 7 (see Fig. 4).

Note that the strip's area $180 \mathrm{deg}^{2}$ corresponds to diameter of the equivalent circular field of view $2 w_{e} \simeq 15.1^{\circ}$, so the expected survey speed is very high. In particular, at the exposure time $20 \mathrm{sec}$ and dead time 5 sec we obtain $S \simeq 7 \mathrm{deg}^{2} / \mathrm{sec}$; at such a speed, the sky region of area $10^{4} \mathrm{deg}^{2}$ will be examined in less than half an hour.

\section{$5 \quad$ Sky survey rate}

Along with a number of standard parameters of telescopes, it would be useful to have a parameter, giving an idea of the efficiency of the telescope just as a survey tool. To date, such a parameter widely used is the etendue $E \equiv \pi w^{2} \cdot \pi D_{e}^{2} / 4$, a product of the observed sky area $\left(\operatorname{deg}^{2}\right)$ and the effective area of the telescope aperture $\left(\mathrm{m}^{2}\right)$. The inadequacy of this measure is evident from the fact that $E$ does not take into account the quality of images provided by survey telescope. However, there is no doubt that the better angular resolution we can reach, the higher is the efficiency of the survey. An adequate measure of survey efficiency, the sky survey rate $\Gamma$, was proposed by V.V. Biryukov and the author (Terebizh 2011). In the case when the dead time is much less than the exposure time, the sky survey rate is proportional to the ratio of the observed sky area $\pi w^{2}$ to the exposure time, needed to achieve the required $S / N$ value. It is not difficult to show that parameter defined in this way is

$$
\Gamma \equiv \frac{\pi w^{2} \cdot \pi D_{e}^{2} / 4}{\Delta^{2}}=E / \Delta^{2}
$$

where $\Delta$ is the so called delivered image quality, measured in angular units. For our purpose, it is sufficient to accept

$$
\Delta=\sqrt{\beta_{\text {atm }}^{2}+\beta_{\text {tel }}^{2}+\left(p^{\prime \prime}\right)^{2}} .
$$


In essence, $\Gamma$ is the product of the number of resolution elements in the observed region of the sky and the effective area of the telescope's aperture 2 . For practical needs a convenient measurement unit of $\Gamma$ is

$$
\text { Herschel } \equiv 1 \mathrm{~m}^{2} \mathrm{deg}^{2} / \operatorname{arcsec}^{2},
$$

named after William Herschel (1738-1822). Hereafter we use an abbreviation $H$.

Values of $\Gamma$ for the systems considered here are given in Table 1. First of all, pay attention to the marked superiority of the telescope No. 1 on the system No. 2, which shows once again the merits of the location the lens corrector in a prime focus.

Then, one might suppose that system No. 4 is significantly more effective than system No. 5, since their apertures do not differ very much, but the field of view of system No. 4 is twice as larger. However, in reality they have the same values of $\Gamma$, which stresses the importance of good image quality for survey telescopes.

As for the lens objective No. 9, its small diameter and a comparatively lower quality of images do not provide large value of $\Gamma$. Nevertheless, very high survey speed makes it quite suitable for observations of rapidly varying objects. For example, at the exposure time $20 \mathrm{sec}$ the survey speed reaches $4.5 \mathrm{deg}^{2} / \mathrm{sec}$, which allows registering of objects brighter than $18.6^{m}$ in the sky area of $10^{4} \mathrm{deg}^{2}$ in just 40 minutes. If necessary, the image quality of the lens can be improved by a slight aspherization of certain surfaces or by using a special glass.

The high efficiency of the $\Omega_{2-3}$-designs No. 7 and No. 8 draws attention. These all-spherical systems offer good images in a large field of view; the secondary passage of light through the input optics enables significant reducing both the aberrations and light obscuration, so the effective aperture diameter is not much inferior to the entrance pupil diameter. As is known, the use of spherical optics significantly mitigates the tolerances in the manufacture and operation of telescopes; ultimately it strongly affects the total cost of the sky survey systems. It can be assumed that these systems will be widely used in future surveys.

As one might expect, the design No. 10 provides the highest survey rate. Continuing discussion in a frame of the example of preceding section, we

\footnotetext{
${ }^{2}$ J. Tonry (2010) proposed a more detailed approach to the evaluation of the survey effectiveness, which includes consideration of the Point Spread Function form and its alignment with the pixels of the detector.
} 
have the field area $180 \mathrm{deg}^{2}$, the equivalent aperture diameter $D_{e}=0.472 \mathrm{~m}$ and the delivered image quality $\Delta=2.41^{\prime \prime}$. According to equation (6), the resulting survey rate $\Gamma \simeq 5.4 \mathrm{H}$, which is almost 3 times greater than the maximum value for the systems in Table 1 .

\section{Concluding remarks}

Since we are primarily interested in the performance of various optical systems, we touch only in passing the issues relating to their manufacturing. For various reasons, the technological challenges are growing rapidly with increasing the speed of a system, say, when the focal ratio becomes less than 1.5. As Table 2 shows, this is not the case for the sample of designs under consideration.

It is also worth adding that each of the designs No. 1-3 contains only one aspheric surface. Thus, the tolerances are quite reasonable for these systems, while for the all-spherical designs No. 4-10 the tolerances are so mild as possible given a system's speed.

We have seen that even a single wide-field telescope with an aperture less than $1 \mathrm{~m}$ and a flat detector, designed and manufactured properly, ensure the registration of objects brighter than $21.5^{m}-22.5^{m}$ in the entire visible hemisphere of the sky within one night. Much faster, but not so deep survey provides a system with the spherical focal surface. Of course, the more extensive and reliable data should come from a hierarchic observational set, comprised of a few telescopes of different types with optimally chosen specifications. Such systems, using previously made telescopes work now effectively, in particular, the Palomar Transient Factory (Law, Kulkarni et al. 2009) and Catalina Real-Time Transient Survey (Djorgovski, Drake et al. 2011). The ATLAS (Asteroid Terrestrial-impact Last Alert System, Tonry 2010), using newly built instruments, is close to completion.

As far as the choice of a telescope's optical scheme substantially depends on a specific task of wide-field observations, there is no preferred option suitable for all occasions. We also need to add the well-known fact that the choice of an optical layout largely depends on the characteristics of the assumed detector of light. However, the two mentioned factors - problem to be solved and the properties of detector, which, of course, one need to add an assessment of the project's cost - almost uniquely define the required optical scheme. 


\section{Acknowledgments}

I thank M.R. Ackermann (University of New Mexico, U.S.A.), V.V. Biryukov (Crimean Astrophysical Observatory), M. Boer (Recherche CNRS ARTEMIS, France), D.A. Kononov (Institute of Astronomy, Russian Academy of Sciences), Yu.A. Petrunin (Telescope Engineering Company, U.S.A.) and J.L. Tonry (Institute for Astronomy, University of Hawaii, U.S.A.) for useful comments to the paper.

\section{References}

[1] Amon M., Rosin S., Jackson B., 1971, Appl. Optics 10, 490

[2] Baker J.G., 1962, U.S. Patent 3,022,708

[3] Busch W., Ceragioli R.C., Stephani W., 2013, Journal of Astronomical History and Heritage 16(2), 107

[4] Blake T., Pearce E., Gregory J.A., Smith A., et al., 2013, AMOS Technical Conference, 57

[5] Djorgovski S.G., Drake A.J., Mahabal A.A. et al., 2011, arXiv:1102.5004v1 [astro-ph.IM] 24 Feb 2011

[6] Hamilton W.F., 1814, Engl. Pat. No. 3781

[7] Hawkins D.G., Linfoot E.H., 1945, Monthly Not. of RAS 105, 334

[8] Hodapp K.W., Kaiser N., Aussel H. et al. 2004, Astron. Nachr. 325, 636

[9] Houghton J.L., 1942, Brit. Patent 546,307

[10] Houghton J.L., 1944, U.S. Patent 2,350,112

[11] Iwert, O., Delabre, B., 2010, Proc. SPIE 7742-27

[12] Iwert, O., Ouelette, D., Lesser M., Delabre, B., 2012, Proc. SPIE 845368

[13] Jeas W.C., 1981, Military Electronics/Countermeasures, November 1981

[14] Korsch D., 1972, Appl. Optics 11, 2986 
[15] Korsch D., 1977, Appl. Optics 16, 2074

[16] Lampton M., Akerlof C., Aldering G. et al., 2002, BAAS 33, 1405

[17] Law N.M., Kulkarni S.R., Dekany R.G. et al., 2009, Publications of the Astronomical Society of the Pacific 121 (886), 1395

[18] Paul M., 1935, Rev. Opt. Theor. Instrum. 14, 169

[19] Press W.H., Teukolsky S.A., Vetterling W.T., Flannery B.P., 1992, Numerical Recipes in C, Cambridge Univ. Press, 500

[20] Richter R., Slevogt H., 1941, Deutsche Patentanmeldung (Zeiss) Z 26 592 IXa 42h, 5.9.1941

[21] Ross F.E., 1935, Astrophys. J. 81, 156

[22] Sampson R.A., 1913, Phil. Trans. R. Soc. London 213, 27

[23] Saunders W., Gillingham P., Smith G., Kent S., Doel P., 2014, Proc. of SPIE 9151, 91511M-1

[24] Schmidt B., 1930, Mitteilungen Hamburger Sternwarte in Bergedorf 7 (36), 15. Reprinted: Selected Papers on Astronomical Optics, D.J. Schroeder (ed.), SPIE Milestone Series 73, 165, 1993

[25] Terebizh V.Yu., 2001, Bull. of the Crimean Astrophysical Obs. 97, 101

[26] Terebizh V.Yu., 2003, Concept Optical Design for a Very WideField Corrector for the Blanco 4-m Telescope, AURA-CTIO Rep. No. C10430A (Terebizh V.Yu. 2004, Astronomy Letters 30, 200).

[27] Terebizh V.Yu., 2007a, arXiv:0710.2165v1 [astro-ph] 11 Oct 2007

[28] Terebizh V.Yu., 2007b, In: "Astronomy 2006: Tradition, Present and Future." St.-Petersburg Univ. Press, 362 (in Russian)

[29] Terebizh V.Yu., 2011, Astron. Nachr. / AN, 332, 714

[30] Terebizh V.Yu., 2015, arXiv:1507.07110v1 [astro-ph.IM] 25 Jul 2015

[31] Terebizh V.Yu., 2016, Astron. Nachr. / AN, In press 
[32] Tonry J., 2010, arXiv:1011.1028v1 [astro-ph.IM] 3 Nov 2010

[33] Wachmann A.A., 1955, Sky and Telescope 15, No.1, 4

[34] Willstrop R.V., 1984, MNRAS 210, 597

[35] Wynne C.G., 1968, Astrophys. J. 152, 675

\section{Appendix. Description of optical systems}

The following is a complete description of the designs, which are included in Table 1 at Nos. 1 - 8 and No. 10. Numerical data are presented in a common format in the Tables $3-11$, the corresponding optical layouts are shown in Fig. 1.

The numbering of surfaces in tables corresponds to the optical path of light. The terms 'aperture' and 'entrance pupil' are considered as equivalent. All distances are given in millimeters. The curvature radii and inter-element distances are presented with the precision required by optical soft packages. We do not round the thicknesses of lenses, because variations of the optical constants of each type of glass at manufacturing will inevitably require minor adjustment of thicknesses.

The sorts of optical glass correspond to those of catalogues by Schott (N...) and Ohara (S-...). In both catalogues, the selection of glass was limited by the maximum melt frequency. Sorts S-BSL7 and N-BK7 are equivalent. The data source for Fused silica is 'The Infrared and Electro-Optical Systems Handbook', Vol. III, Ch. 1.

Since the filter and detector window have zero optical power, their position and thickness can be changed with slight correction of the basic optical layout.

As usual, optical schemes can be scaled up or down. Since image quality is not far from the diffraction limit, the scaling up should be performed along with slight optimization.

The brief designations are the following: $R_{0}$ - paraxial curvature radius, $T$ - distance to the next surface, $D$ - light diameter, FS - fused silica, Stop aperture stop, $\mathrm{SP}$ - stop position, $\mathrm{L}_{k}-\mathrm{k}$-th lens, $\mathrm{Pri}$ - primary mirror, Sec secondary mirror, Obsc - obscuration, $\mathrm{F}$ - filter, $\mathrm{W}$ - window of the detector, Ima - image on the focal surface. 
Table 3: VT-56y design with an aperture of $1.0 \mathrm{~m}$ and $3.5^{\circ}$ field. The effective focal length is $2183 \mathrm{~mm}$. The design waveband is $0.40-0.85 \mu \mathrm{m}$.

\begin{tabular}{cccccc}
\hline $\begin{array}{c}\text { Surf. } \\
\text { No. }\end{array}$ & $\begin{array}{c}\text { Com- } \\
\text { ments }\end{array}$ & $\begin{array}{c}R_{0} \\
(\mathrm{~mm})\end{array}$ & $\begin{array}{c}T \\
(\mathrm{~mm})\end{array}$ & Glass & $\begin{array}{c}D \\
(\mathrm{~mm})\end{array}$ \\
\hline 1 & Stop & $\infty$ & 26.509 & - & 1000.0 \\
2 & Pri & -4708.58 & -1645.73 & Mirror & 1000.0 \\
3 & $\mathrm{~L}_{1}$ & -419.235 & -45.0 & N-BK7 & 391.6 \\
4 & & -1199.06 & -101.877 & - & 384.5 \\
5 & $\mathrm{~L}_{2}$ & -504.582 & -24.40 & FS & 304.5 \\
6 & & -244.457 & -61.976 & - & 274.4 \\
7 & $\mathrm{~L}_{3}$ & 1854.23 & -34.50 & N-LAK8 & 267.9 \\
8 & & -344.778 & -23.148 & - & 255.9 \\
9 & $\mathrm{~L}_{4}$ & -2822.80 & -20.0 & S-PHM52 & 256.2 \\
10 & & 757.208 & -187.357 & - & 256.9 \\
11 & $\mathrm{~L}_{5}$ & -406.165 & -42.40 & S-FPL53 & 252.7 \\
12 & & 496.992 & -148.232 & - & 251.4 \\
13 & $\mathrm{~F}$ & $\infty$ & -7.0 & N-BK7 & 157.6 \\
14 & & $\infty$ & -18.0 & - & 155.1 \\
15 & $\mathrm{~W}$ & $\infty$ & -6.0 & FS & 144.8 \\
16 & & $\infty$ & -14.0 & - & 142.5 \\
17 & Ima & $\infty$ & - & - & 134.5 \\
\hline
\end{tabular}

Notes to Table 3:

Conic constant of the primary mirror is -1.506709 , all other surfaces are spheres.

Light obscuration corresponds to the round screen of diameter $392 \mathrm{~mm}$. 
Table 4: VT-112m design with an aperture of $1.0 \mathrm{~m}$ and $3.5^{\circ}$ field. The effective focal length is $2189 \mathrm{~mm}$. The design waveband is $0.45-0.85 \mu \mathrm{m}$.

\begin{tabular}{cccccc}
\hline $\begin{array}{c}\text { Surf. } \\
\text { No. }\end{array}$ & $\begin{array}{c}\text { Com- } \\
\text { ments }\end{array}$ & $\begin{array}{c}R_{0} \\
(\mathrm{~mm})\end{array}$ & $\begin{array}{c}T \\
(\mathrm{~mm})\end{array}$ & Glass & $\begin{array}{c}D \\
(\mathrm{~mm})\end{array}$ \\
\hline 1 & Stop & $\infty$ & 28.944 & - & 1000.0 \\
2 & Pri & -4311.49 & -1139.21 & Mirror & 1000.0 \\
3 & Sec & -11457.9 & 601.547 & Mirror & 549.5 \\
4 & $\mathrm{~L}_{1}$ & 342.969 & 32.0 & N-BK7 & 350.0 \\
5 & & 239.293 & 44.296 & - & 322.7 \\
6 & $\mathrm{~L}_{2}$ & 622.257 & 32.0 & N-LAK9 & 322.6 \\
7 & & 20122.9 & 130.451 & - & 320.0 \\
8 & $\mathrm{~L}_{3}$ & 766.109 & 26.0 & N-LAK10 & 265.7 \\
9 & & 249.604 & 102.599 & - & 248.2 \\
10 & $\mathrm{~L}_{4}$ & 363.158 & 70.0 & S-FPL53 & 263.8 \\
11 & & -294.997 & 94.073 & - & 262.1 \\
12 & $\mathrm{~L}_{5}$ & -595.004 & 17.360 & N-LAK14 & 178.9 \\
13 & & -11372.6 & 30.005 & - & 172.6 \\
14 & $\mathrm{~F}$ & $\infty$ & 5.0 & N-BK7 & 158.6 \\
15 & & $\infty$ & 25.0 & - & 157.1 \\
16 & $\mathrm{~W}$ & $\infty$ & 4.0 & FS & 145.5 \\
17 & & $\infty$ & 21.0 & - & 144.2 \\
18 & Ima & $\infty$ & - & - & 134.5 \\
\hline
\end{tabular}

Notes to Table 4:

Conic constant of the primary mirror is -1.495564 , all other surfaces are spheres. Light obscuration corresponds to the round screen of diameter $560 \mathrm{~mm}$. 
Table 5: VT-110f design with an aperture of $500 \mathrm{~mm}$ and $7.5^{\circ}$ field. The effective focal length is $1026 \mathrm{~mm}$. The design waveband is $0.42-0.82 \mu \mathrm{m}$.

\begin{tabular}{cccccc}
\hline $\begin{array}{c}\text { Surf. } \\
\text { No. }\end{array}$ & $\begin{array}{c}\text { Com- } \\
\text { ments }\end{array}$ & $\begin{array}{c}R_{0} \\
(\mathrm{~mm})\end{array}$ & $\begin{array}{c}T \\
(\mathrm{~mm})\end{array}$ & Glass & $\begin{array}{c}D \\
(\mathrm{~mm})\end{array}$ \\
\hline 1 & $\mathrm{~L}_{1}$ & $\infty$ & 25.142 & FS & 502.2 \\
2 & Stop & $\infty$ & 1472.789 & - & 500.0 \\
3 & Pri & -2336.261 & -979.429 & Mirror & 680.2 \\
4 & $\mathrm{~L}_{1}$ & -376.877 & -24.832 & SF10 & 225.0 \\
5 & & 9297.360 & -2.035 & - & 221.3 \\
6 & $\mathrm{~L}_{2}$ & 2346.990 & -18.356 & N-LAF7 & 221.2 \\
7 & & -210.890 & -3.828 & - & 198.8 \\
8 & $\mathrm{~L}_{3}$ & -196.733 & -45.723 & S-FPL53 & 198.2 \\
9 & & 946.597 & -40.557 & - & 192.6 \\
10 & $\mathrm{~F}$ & $\infty$ & -4.0 & N-BK7 & 160.6 \\
11 & & $\infty$ & -16.0 & - & 158.8 \\
12 & $\mathrm{~W}$ & $\infty$ & -4.0 & FS & 147.6 \\
13 & & $\infty$ & -16.0 & - & 145.7 \\
14 & $\mathrm{Ima}$ & $\infty$ & - & - & 134.5 \\
\hline
\end{tabular}

Notes to Table 5:

Obscuration on surface No. 2 of diameter $250.0 \mathrm{~mm}$.

Surface No. 2 is even asphere with $A_{2}=-2.08187 \cdot 10^{-5}, A_{4}=4.811443 \cdot 10^{-11}$. All other surfaces are spheres. 
Table 6: VT-78e design with an aperture of $400 \mathrm{~mm}$ and $10^{\circ}$ field. The effective focal length is $764 \mathrm{~mm}$. The design waveband is $0.45-0.85 \mu \mathrm{m}$.

\begin{tabular}{cccccc}
\hline $\begin{array}{c}\text { Surf. } \\
\text { No. }\end{array}$ & $\begin{array}{c}\text { Com- } \\
\text { ments }\end{array}$ & $\begin{array}{c}R_{0} \\
(\mathrm{~mm})\end{array}$ & $\begin{array}{c}T \\
(\mathrm{~mm})\end{array}$ & Glass & $\begin{array}{c}D \\
(\mathrm{~mm})\end{array}$ \\
\hline 1 & $\mathrm{~L}_{1}$ & 1077.981 & 59.681 & N-BK7 & 484.8 \\
2 & & -1221.197 & 78.931 & - & 482.6 \\
3 & $\mathrm{~L}_{2}$ & -1251.780 & 37.591 & N-BK7 & 425.4 \\
4 & & 2367.109 & 39.113 & - & 406.3 \\
5 & $\mathrm{~L}_{3}$ & -694.206 & 39.247 & N-BK7 & 406.3 \\
6 & & -1770.605 & 262.603 & - & 405.4 \\
7 & Stop & $\infty$ & 15.726 & - & 365.3 \\
8 & Pri & -1072.895 & -278.328 & Mirror & 365.3 \\
9 & Sec & -1770.605 & 279.256 & Mirror & 239.0 \\
10 & $\mathrm{~L}_{4}$ & 398.439 & 40.0 & N-SK10 & 154.0 \\
11 & $\mathrm{~L}_{5}$ & -395.695 & 25.0 & P-LAF37 & 154.0 \\
12 & & -36384.17 & 29.359 & - & 154.0 \\
13 & $\mathrm{~F}$ & $\infty$ & 5.0 & N-BK7 & 154.7 \\
14 & & $\infty$ & 25.0 & - & 153.3 \\
15 & $\mathrm{~W}$ & $\infty$ & 5.0 & FS & 142.5 \\
16 & & $\infty$ & 15.0 & - & 141.0 \\
17 & Ima & $\infty$ & - & - & 134.5 \\
\hline
\end{tabular}

Notes to Table 6 :

Obscuration on surface No. 6 of diameter $188.0 \mathrm{~mm}$.

All surfaces are spheres. 
Table 7: VT-77i design with an aperture of $500 \mathrm{~mm}$ and $5.0^{\circ}$ field. The effective focal length is $1538 \mathrm{~mm}$. The design waveband is $0.45-0.85 \mu \mathrm{m}$.

\begin{tabular}{cccccc}
\hline $\begin{array}{c}\text { Surf. } \\
\text { No. }\end{array}$ & $\begin{array}{c}\text { Com- } \\
\text { ments }\end{array}$ & $\begin{array}{c}R_{0} \\
(\mathrm{~mm})\end{array}$ & $\begin{array}{c}T \\
(\mathrm{~mm})\end{array}$ & Glass & $\begin{array}{c}D \\
(\mathrm{~mm})\end{array}$ \\
\hline 1 & $\mathrm{~L}_{1}$ & 2667.080 & 44.0 & N-BK7 & 527.0 \\
2 & & 14019.45 & 278.784 & - & 522.8 \\
3 & $\mathrm{~L}_{2}$ & -1843.524 & 40.0 & N-BK7 & 478.0 \\
4 & Stop & -4428.837 & 646.791 & - & 476.9 \\
5 & Pri & -2430.967 & -646.791 & Mirror & 534.7 \\
6 & Sec & -4428.837 & 621.791 & Mirror & 310.1 \\
7 & $\mathrm{~L}_{3}$ & 802.225 & 45.0 & N-KZFS4 & 177.3 \\
8 & $\mathrm{~L}_{4}$ & 174.767 & 36.0 & N-LAK12 & 164.3 \\
9 & & 1702.637 & 41.971 & - & 160.0 \\
10 & $\mathrm{~F}$ & $\infty$ & 5.0 & N-BK7 & 145.9 \\
11 & & $\infty$ & 10.0 & - & 144.8 \\
12 & $\mathrm{~W}$ & $\infty$ & 3.0 & FS & 141.2 \\
13 & & $\infty$ & 17.0 & - & 140.5 \\
14 & $\mathrm{Ima}$ & $\infty$ & - & - & 134.5 \\
\hline
\end{tabular}

Notes to Table 7:

Obscuration on surface No. 4 of diameter $244.0 \mathrm{~mm}$.

All surfaces are spheres. 
Table 8: VT-98v design with an aperture of $500 \mathrm{~mm}$ and $7^{\circ}$ field. The effective focal length is $1093 \mathrm{~mm}$. The design waveband is $0.45-0.85 \mu \mathrm{m}$.

\begin{tabular}{cccccc}
\hline $\begin{array}{c}\text { Surf. } \\
\text { No. }\end{array}$ & $\begin{array}{c}\text { Com- } \\
\text { ments }\end{array}$ & $\begin{array}{c}R_{0} \\
(\mathrm{~mm})\end{array}$ & $\begin{array}{c}T \\
(\mathrm{~mm})\end{array}$ & Glass & $\begin{array}{c}D \\
(\mathrm{~mm})\end{array}$ \\
\hline 1 & $\mathrm{~L}_{1}$ & 1961.024 & 65.083 & FS & 582.3 \\
2 & & -1654.074 & 60.033 & - & 579.1 \\
3 & $\mathrm{~L}_{2}$ & -1858.116 & 39.028 & FS & 550.2 \\
4 & & 79341.23 & 405.393 & - & 538.4 \\
5 & Stop & $\infty$ & 22.105 & - & 421.8 \\
6 & $\mathrm{~L}_{3}$ & -1086.270 & 45.923 & FS & 421.8 \\
7 & Pri & -2128.737 & -45.923 & Mirror & 429.1 \\
8 & $\mathrm{~L}_{3}$ & -1086.270 & -427.499 & - & 414.0 \\
9 & Sec & 79341.23 & 515.142 & Mirror & 323.1 \\
10 & $\mathrm{~L}_{4}$ & 305.528 & 23.460 & FK3 & 200.0 \\
11 & & -3053.207 & 30.473 & - & 198.2 \\
12 & $\mathrm{~L}_{5}$ & -661.789 & 16.418 & S-TIM22 & 182.7 \\
13 & & -768.247 & 28.810 & - & 178.7 \\
14 & $\mathrm{~F}$ & $\infty$ & 7.0 & N-BK7 & 159.5 \\
15 & & $\infty$ & 23.0 & - & 156.9 \\
16 & $\mathrm{~W}$ & $\infty$ & 10.0 & FS & 143.9 \\
17 & & $\infty$ & 10.0 & - & 140.2 \\
18 & Ima & $\infty$ & - & - & 134.5 \\
\hline
\end{tabular}

Notes to Table 8:

Obscuration on surface No. 4 of diameter $270.0 \mathrm{~mm}$.

All surfaces are spheres. 
Table 9: VT-102j design with an aperture of $525 \mathrm{~mm}$ and $7.0^{\circ}$ field. The effective focal length is $1094 \mathrm{~mm}$. The design waveband is $0.43-0.85 \mu \mathrm{m}$.

\begin{tabular}{cccccc}
\hline $\begin{array}{c}\text { Surf. } \\
\text { No. }\end{array}$ & $\begin{array}{c}\text { Com- } \\
\text { ments }\end{array}$ & $\begin{array}{c}R_{0} \\
(\mathrm{~mm})\end{array}$ & $\begin{array}{c}T \\
(\mathrm{~mm})\end{array}$ & Glass & $\begin{array}{c}D \\
(\mathrm{~mm})\end{array}$ \\
\hline 1 & Stop & $\infty$ & 150.0 & - & 525.0 \\
2 & Obsc & $\infty$ & 64.192 & - & 240.0 \\
3 & $\mathrm{~L}_{1}$ & 4516.956 & 61.791 & FS & 552.3 \\
4 & & -4110.190 & 828.460 & - & 554.3 \\
5 & $\mathrm{~L}_{2}$ & -1249.675 & 53.0 & FS & 558.6 \\
6 & Pri & -2262.803 & -53.0 & Mirror & 568.4 \\
7 & $\mathrm{~L}_{2}$ & -1249.675 & -828.460 & - & 544.8 \\
8 & $\mathrm{~L}_{1}$ & -4110.190 & -61.791 & FS & 285.2 \\
9 & & 4516.956 & -64.192 & - & 271.5 \\
10 & $\mathrm{~L}_{3}$ & -419.927 & -50.0 & S-PHM52 & 239.4 \\
11 & & 1169.041 & -0.20 & - & 226.8 \\
12 & $\mathrm{~L}_{4}$ & 1167.046 & -44.897 & S-LAH55 & 226.6 \\
13 & & -16723.57 & -63.966 & - & 207.4 \\
14 & $\mathrm{~F}$ & $\infty$ & -5.0 & S-BSL7 & 161.4 \\
15 & & $\infty$ & -15.0 & - & 159.1 \\
16 & $\mathrm{~W}$ & $\infty$ & -3.0 & FS & 148.3 \\
17 & & $\infty$ & -17.0 & - & 146.8 \\
18 & Ima & $\infty$ & & - & 134.5 \\
\hline
\end{tabular}

Notes to Table 9:

Obscuration on surface No. 2 of diameter $240.0 \mathrm{~mm}$.

All surfaces are spheres. 
Table 10: VT-60g design with an aperture of $700 \mathrm{~mm}$ and $7.0^{\circ}$ field. The effective focal length is $1091 \mathrm{~mm}$. The design waveband is $0.45-0.85 \mu \mathrm{m}$.

\begin{tabular}{cccccc}
\hline $\begin{array}{c}\text { Surf. } \\
\text { No. }\end{array}$ & $\begin{array}{c}\text { Com- } \\
\text { ments }\end{array}$ & $\begin{array}{c}R_{0} \\
(\mathrm{~mm})\end{array}$ & $\begin{array}{c}T \\
(\mathrm{~mm})\end{array}$ & Glass & $\begin{array}{c}D \\
(\mathrm{~mm})\end{array}$ \\
\hline 1 & Obsc & $\infty$ & 189.472 & - & 238.0 \\
2 & $\mathrm{~L}_{1}$ & 2451.467 & 55.0 & FS & 730.3 \\
3 & & -11435.65 & 219.271 & - & 726.4 \\
4 & Stop & $\infty$ & 157.498 & - & 660.2 \\
5 & $\mathrm{~L}_{2}$ & -1425.622 & 55.0 & FS & 656.7 \\
6 & & -1181.598 & 356.662 & - & 662.5 \\
7 & $\mathrm{~L}_{3}$ & -991.351 & 55.0 & FS & 634.6 \\
8 & Pri & -2113.002 & -55.0 & Mirror & 647.4 \\
9 & $\mathrm{~L}_{3}$ & -991.351 & -356.662 & - & 610.7 \\
10 & $\mathrm{~L}_{2}$ & -1181.598 & -55.0 & FS & 480.6 \\
11 & & -1425.622 & -157.498 & - & 461.1 \\
12 & $\mathrm{SP}$ & $\infty$ & -219.271 & - & 200.7 \\
13 & $\mathrm{~L}_{1}$ & -11435.65 & -55.0 & FS & 306.7 \\
14 & & 2451.467 & -119.919 & - & 292.0 \\
15 & $\mathrm{~L}_{4}$ & -330.389 & -35.210 & N-BK7 & 220.0 \\
16 & & 619.249 & -2.274 & - & 216.1 \\
17 & $\mathrm{~L}_{5}$ & 592.307 & -32.069 & N-BAK4 & 213.5 \\
18 & & -4564.917 & -20.998 & - & 190.9 \\
19 & $\mathrm{~F}$ & $\infty$ & -5.0 & N-BK7 & 173.9 \\
20 & & $\infty$ & -25.0 & - & 171.2 \\
21 & $\mathrm{~W}$ & $\infty$ & -5.0 & N-BK7 & 149.9 \\
22 & & $\infty$ & -15.0 & - & 147.3 \\
23 & Ima & $\infty$ & & & 134.5 \\
\hline
\end{tabular}

Notes to Table 10:

Obscuration on surface No. 1 of diameter $238.0 \mathrm{~mm}$.

All surfaces are spheres. 
Table 11: VT-119j design with an aperture of $500 \mathrm{~mm}$ and $45^{\circ}$ field of view. The effective focal length is $1358 \mathrm{~mm}$. The design waveband is $0.45-0.85 \mu \mathrm{m}$.

\begin{tabular}{cccccc}
\hline $\begin{array}{c}\text { Surf. } \\
\text { No. }\end{array}$ & $\begin{array}{c}\text { Com- } \\
\text { ments }\end{array}$ & $\begin{array}{c}R_{0} \\
(\mathrm{~mm})\end{array}$ & $\begin{array}{c}T \\
(\mathrm{~mm})\end{array}$ & Glass & $\begin{array}{c}D \\
(\mathrm{~mm})\end{array}$ \\
\hline 1 & $\mathrm{~L}_{1}$ & 2279.917 & 95.0 & FS & 1000.0 \\
2 & & 2456.517 & 522.273 & - & 940.2 \\
3 & $\mathrm{~L}_{2}$ & -4963.27 & 74.860 & FS & 536.4 \\
4 & & -4531.56 & 0.001 & - & 496.9 \\
5 & Stop & $\infty$ & 746.412 & - & 491.0 \\
6 & $\mathrm{~L}_{3}$ & -1044.83 & 101.742 & FS & 1004.7 \\
7 & & -1075.10 & 75.840 & - & 1081.8 \\
8 & $\mathrm{~L}_{4}$ & -912.495 & 105.015 & FS & 1109.1 \\
9 & & -1039.24 & 1637.765 & - & 1209.4 \\
10 & Pri & -2788.70 & -1431.74 & Mirror & 2500.0 \\
11 & Ima & -1328.68 & - & - & 1039.0 \\
\hline
\end{tabular}

Note to Table 11:

All surfaces are spheres. 